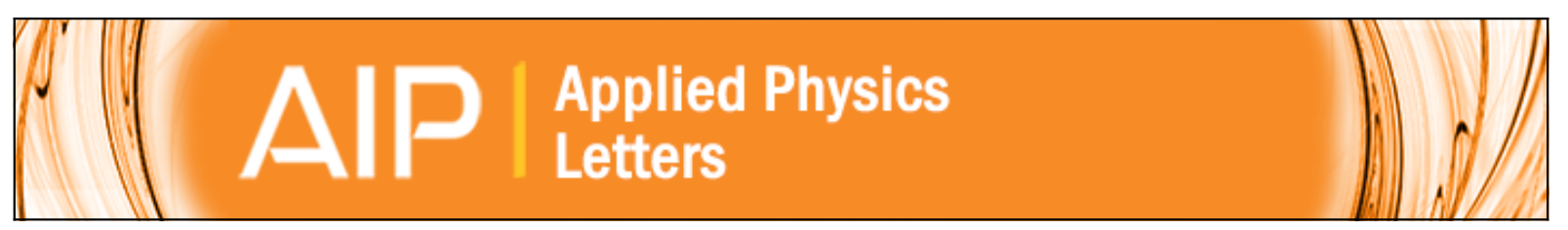

\title{
Electronic and quantum phase coherence properties of bismuth thin films
}

M. Rudolph and J. J. Heremans

Citation: Applied Physics Letters 100, 241601 (2012); doi: 10.1063/1.4729035

View online: http://dx.doi.org/10.1063/1.4729035

View Table of Contents: http://scitation.aip.org/content/aip/journal/apl/100/24?ver=pdfcov

Published by the AIP Publishing

AlP Re-register for Table of Content Alerts 


\title{
Electronic and quantum phase coherence properties of bismuth thin films
}

\author{
M. Rudolph ${ }^{\text {a) }}$ and J. J. Heremans ${ }^{\text {b) }}$ \\ Department of Physics, Virginia Tech, Blacksburg, Virginia 24061, USA
}

(Received 6 April 2012; accepted 24 May 2012; published online 11 June 2012)

\begin{abstract}
We present a method to deposit bulk-like Bi films by thermal evaporation and study the electrical, quantum coherence, and physical properties. A two stage growth procedure was found to optimize the film properties, with an initial wetting layer deposited at lower temperature followed by an active layer at higher temperature. Transport measurements indicate carrier properties comparable to molecular beam epitaxial films and display weak-antilocalization, from which the quantum phase coherence lengths are deduced. A $76 \mathrm{~nm}$ film is found to optimally exhibit both bulk-like Bi characteristics and the 2-dimensional quantum coherence properties desired for Bi-based quantum devices. (C) 2012 American Institute of Physics. [http://dx.doi.org/10.1063/1.4729035]
\end{abstract}

Bulk bismuth, with its highly anisotropic Fermi surfaces, was historically a gateway for discovering quantum effects in the solid state. ${ }^{1-4}$ The electrons support a Diraclike spectrum with low density, high mobility $\left(>5000 \mathrm{~m}^{2} / \mathrm{Vs}\right)$, long mean free path, and Fermi wavelength, and the carriers possess strong spin-orbit interaction (SOI). ${ }^{5}$ In addition, the $\mathrm{Bi}(111)$ surface (rhombohedral indexing) supports metallic states with pronounced Rashbatype SOI. ${ }^{6,7}$ Harnessing the quantum properties of bulk Bi in nanodevices opens the door to studying unique systems and interactions. However, growing high quality thin films of $\mathrm{Bi}$ has been challenging, 8,9 and the study of patterned $\mathrm{Bi}$ nanostructures has been sparse, ${ }^{10,11}$ so efforts to produce Bi thin films with bulk-like properties are necessary.

While quantum properties of disordered Bi films have been studied, we focus on large grain films to retain the desired properties of bulk $\mathrm{Bi}$, which have not been extensively studied. We will discuss an optimized method for growing high quality $\mathrm{Bi}$ films by first growing a wetting layer to minimize substrate effects. Then, we will present our data on the electrical properties of films of various thicknesses. At low temperatures, transport measurements display weak-antilocalization (WAL) in the thinner films due to coherent interference of time reversed electron paths. From the WAL, we extract the phase coherence length $L_{\phi}$ and discuss its dependence on temperature $T$ and film thickness.

Bismuth films were grown onto amorphous $\mathrm{SiO}_{2}$ substrates by thermal evaporation of $99.999 \%$ pure $\mathrm{Bi}$ in vacuum better than $10^{-8}$ Torr. The substrates were heated in vacuum to $250^{\circ} \mathrm{C}$ for $18 \mathrm{~h}$. The substrate temperature was controlled during the film growth, with the evaporation rate and thickness being ascertained by a quartz balance thickness monitor.

Hall bar structures were etched into the films using standard photolithography techniques and wet chemical etching with $\mathrm{H}_{2} \mathrm{O}_{2}: \mathrm{H}_{2} \mathrm{SO}_{4}: \mathrm{H}_{2} \mathrm{O}$ solution. Measurements of the film thickness, surface roughness, grain size, and Hall bar dimensions were performed by atomic force microscopy (AFM). Wood's metal solder provided Ohmic contacts to the

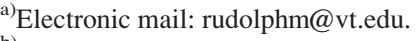

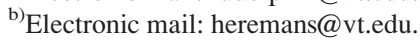

Hall bar structures. The magnetotransport coefficients of the films were measured from room temperature to $2 \mathrm{~K}$.

$\mathrm{Bi}$ has strong cohesion, so film growth begins with island formation, even for Bi grown on lattice matched substrates by molecular beam epitaxy (MBE). ${ }^{8,12}$ In addition, high quality Bi films must be grown at temperatures near the melting point of $\mathrm{Bi}\left(271^{\circ} \mathrm{C}\right)$ to increase the grain size and reduce defects. However, high temperatures further hinder the adhesion of $\mathrm{Bi}$ to the substrate. It has moreover been shown that MBE deposition of $\mathrm{Bi}$ at $150 \mathrm{~K}$ improves initial film growth but leads to disordered films at higher thickness. ${ }^{13}$ To overcome these obstacles, we explore the initial room temperature growth properties of $\mathrm{Bi}$, with as objective to identify if the initial growth at room temperature provides a suitable wetting layer on which a high quality film can subsequently be deposited.

A series of films with thicknesses 10, 15, 20, 25, and $30 \mathrm{~nm}$ were grown at $24{ }^{\circ} \mathrm{C}$ at a rate of $0.05 \mathrm{~nm} / \mathrm{s}$. The thickness is obtained from the quartz balance. Figure 1(a) compares the quartz balance thickness with the thickness obtained by AFM analysis, with the dashed line representing a one to one correspondence between the two measurements. For depositions of less than $20 \mathrm{~nm}$, the AFM thickness is constant at $20 \mathrm{~nm}$, indicating that the initial growth occurs by island formation, with a minimum island dimension of $20 \mathrm{~nm}$. As more $\mathrm{Bi}$ is deposited, the islands grow laterally and the AFM thickness does not change. Only once the islands have merged and the substrate is fully covered does the film grow vertically. Figure 1(b) compares the RMS surface roughness as found by AFM with the film thickness. The roughness does not begin to significantly increase until $20 \mathrm{~nm}$, supporting the conclusion that vertical film growth does not commence until a $20 \mathrm{~nm}$ film is formed. From this analysis, a $20 \mathrm{~nm}$ thick film is an optimal wetting layer, covering the substrate sufficiently.

A series of films with thickness of $30 \mathrm{~nm}$ were grown onto substrates at $24,100,150,200$, and $250{ }^{\circ} \mathrm{C}$ to determine the optimum temperature for the wetting layer. As presented in Figs. 1(c) and 1(d), the maximum grain height (from the substrate) and the roughness of the films monotonically increases with growth temperature. The maximum grain height displays a minimum at $100{ }^{\circ} \mathrm{C}$, and we hence conclude that $100{ }^{\circ} \mathrm{C}$ represents the highest substrate temperature yielding a smooth wetting layer. 


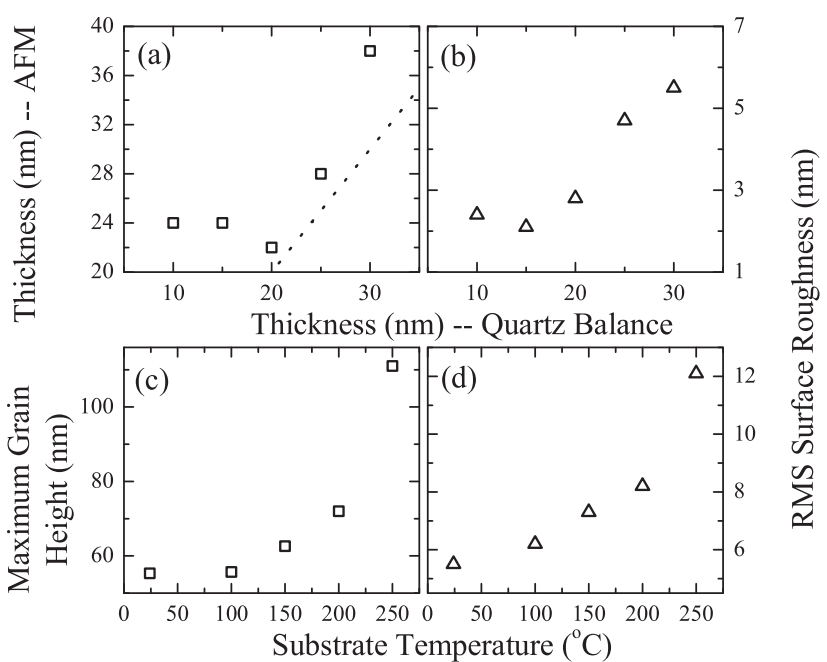

FIG. 1. Physical properties of the initial growth of Bi films. The in-situ measurement of the thickness is compared to the AFM thickness (a) and the surface roughness of the film (b). Films grown at different substrate temperatures are compared to the maximum grain height (c) and surface roughness (d).

To grow high quality films, we implement a two stage growth procedure (Fig. 2(a)). First, a wetting layer of $20 \mathrm{~nm}$ was deposited at $100{ }^{\circ} \mathrm{C}$ and $0.05 \mathrm{~nm} / \mathrm{s}$. The substrate was then further heated to just below the melting temperature of $\mathrm{Bi}\left(\approx 260^{\circ} \mathrm{C}\right)$, at which varying thicknesses were deposited at $0.05 \mathrm{~nm} / \mathrm{s}$. The total thicknesses $t$ determined by the quartz balance (AFM) of the series of films were 40 (47), 50 (55), 70 (76), 100 (108), and $200 \mathrm{~nm}$ (225). Henceforth indicated thicknesses result from AFM measurements. The x-ray diffraction spectrum of a $76 \mathrm{~nm}$ film is displayed in Fig. 2(b), showing the high structural quality of the films. Along with a $\mathrm{Si}(004)$ peak from the substrate, only the $\mathrm{Bi}(003)$ family of peaks are present, indicating oriented film growth with the $\mathrm{Bi}(001)$ direction (trigonal axis) oriented normal to the substrate. The in-plane orientation of the grains is random.

The resistivity decreases asymptotically as the film thickness is increased, as shown in Fig. 3(a). We model the

(a)

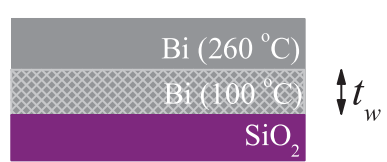

(b)

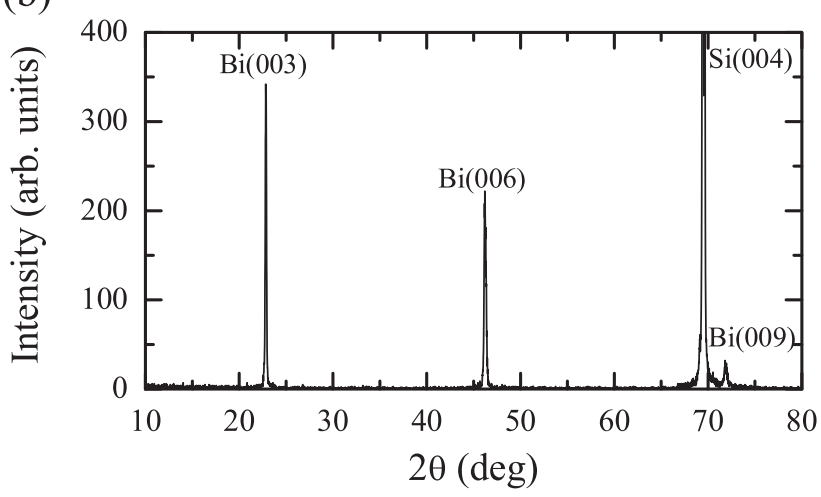

FIG. 2. (a) Schematic of the two stage growth with wetting layer thickness $t_{w}$. (b) X-ray diffraction spectrum of a $76 \mathrm{~nm}$ film indicating high quality growth along the $\mathrm{Bi}(001)$ direction.
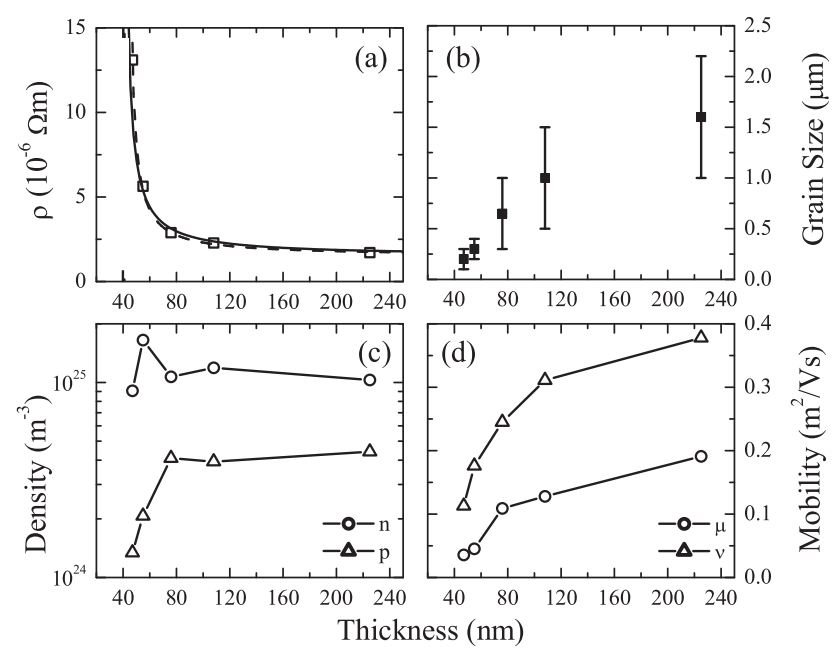

FIG. 3. The room temperature resistivity with fits to Eq. (1) without (solid) and with (dashed) a surface oxide layer (a). The grain size (b), carrier densities (c), and carrier mobilities (d) all increase with film thickness at room temperature.

resistivity as originating from two parallel conducting channels, namely the wetting layer and the bulk-like active layer, and obtain

$$
\rho(t)=\frac{\rho_{b}}{1-t_{w} / t\left(1-\rho_{b} / \rho_{w}\right)}
$$

where $\rho_{w}$ and $\rho_{b}$ are the resistivities of the wetting layer and the active layer, respectively, and $t_{w}$ is the thickness of the wetting layer. The solid line in Fig. 3(a) is achieved with $\rho_{b}=1.5 \times 10^{-6} \Omega \mathrm{m}, \rho_{w}=1.5 \times 10^{-4} \Omega \mathrm{m}$, and $t_{w}=40 \mathrm{~nm}$. Single crystal bulk Bi has a comparable yet slightly lower resistivity $\left(1.1 \times 10^{-6} \Omega \mathrm{m}\right)$ than the active layer, indicating the high quality but still polycrystalline nature of our films' active layer. From an independent measurement, the resistivity of a $20 \mathrm{~nm}$ film is $2.2 \times 10^{-4} \Omega \mathrm{m}$, comparable with $\rho_{w}$. Note that the actual deposited thickness of the wetting layer is $20 \mathrm{~nm}$, indicating that another $20 \mathrm{~nm}$ of $\mathrm{Bi}$ is required to relax the wetting layer to a bulk-like film. The dashed line takes into account the presence of an insulating surface oxide, where we set $t \rightarrow t-t_{o}$, with $t_{o}$ being the oxide thickness. From the fitting, we obtain $t_{w}=31 \mathrm{~nm}$ and $t_{o}=12 \mathrm{~nm}$. These values are consistent with $10 \mathrm{~nm}$ surface oxidation in Bi evidenced by transmission electron microscopy. ${ }^{14}$

Figures 3(b)-3(d) show the grain size, carrier density, and carrier mobility (at room temperature) increasing with film thickness. The grain size increases significantly above $76 \mathrm{~nm}$, where also the resistivity approaches the bulk value. Fitting a 2-carrier model to magnetoresistance and Hall measurements, we find the electron and hole densities and mobilities. As the thickness increases, the electron and hole densities compensate, as expected for neutral bulk Bi. The densities do not change above $76 \mathrm{~nm}$, and the mobilities monotonically increase with film thickness. While the hole mobilities are comparable to values for Bi films grown by $\mathrm{MBE}^{8}$ the electron mobilities are $\sim 4$ times smaller. Due to a higher electron density likely from lattice defects, our films' overall resistivities approach resistivities measured on epitaxial films. Our results indicate that, on a $\mathrm{SiO}_{2}$ substrate, a $76 \mathrm{~nm}$ film is the thinnest Bi film for which the wetting 
layer does not have a large influence on the electrical properties.

We measure the quantum coherence lengths by WAL. WAL is a quantum coherent transport phenomenon due to the destructive interference of time reversed paths and is sensitive to the phase coherence length $L_{\phi}$ and spin coherence length $L_{s o}$. The coherence can be destroyed by breaking time reversal symmetry via an applied magnetic field $B$ and by inducing inelastic scattering via increasing the temperature. The decay of the WAL signal can be tracked with respect to $B$ and $T$ and compared to the theory for 2-dimensional (2D) diffusive systems with heavy element $\mathrm{SOI}^{15,16}$

$$
\begin{aligned}
& \Delta \rho(B, T)= \frac{e^{2} \rho^{2}}{2 \pi^{2} \hbar t}\left[\frac{1}{2} F\left(\frac{H_{\phi}(T)}{B}+\frac{4 H_{s o}}{3 B}\right)\right. \\
&\left.-\frac{3}{2} F\left(\frac{H_{\phi}(T)}{B}\right)+F\left(\frac{H_{0}}{B}+\frac{H_{s o}}{B}\right)\right], \\
& \Delta \rho(B=0, T)=\frac{e^{2} p \rho^{2}}{4 \pi^{2} \hbar t} \ln \left(T / T_{0}\right),
\end{aligned}
$$

where $F(x)=\Psi(1 / 2+x)-\ln (x)$ and $\Psi(x)$ is the digamma function; $H_{0, \phi, s o}=\hbar /\left(4 e L_{0, \phi . s o}^{2}\right)$ with $0, \phi$, so denoting momentum, phase, and spin-orbit scattering, respectively; $p$ represents the exponent in the quantum phase decoherence power law $L_{\phi} \sim T^{-p / 2}$; and $T_{0}$ is the characteristic temperature for the onset of decoherence.

At $T<20 \mathrm{~K}$, WAL is present in the $B$ - and the $T$ dependence of the resistivity of the three thinnest films, depicted in Figs. 4(a) and 4(b) with the best fits (solid lines) to Eqs. (2) and (3), respectively. From the $B$-dependence, $L_{\phi}$ and $L_{s o}$ are extracted, displayed in Figs. 4(c) and 4(d). The phase decoherence exponent is $p=2.2 \pm 0.1$ for all three films, consistent with dominant phonon scattering, as expected at these temperatures. The values of $p$ extracted by applying Eq. (3) to Fig. 4(b) are $p=1.8,2.1$, and 1.3 for the 76,55 , and $47 \mathrm{~nm}$ films, respectively. The exponents $p$ for thicker films are consistent with phonon scattering as also obtained from the $B$-dependence. The $47 \mathrm{~nm}$ film shows a deviation, likely due to an uncertainty for thinner films
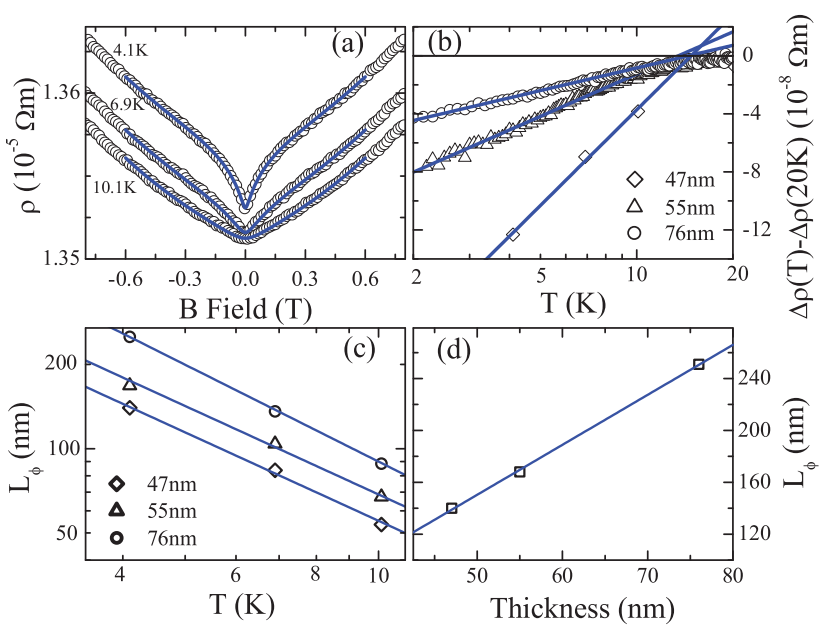

FIG. 4. The enhancement of WAL is evident (a) as function of $B$ for the $55 \mathrm{~nm}$ thick film and (b) as a function of $T$. The extracted phase coherence lengths follow a $T^{-1}$ dependence (c) as well as a linear dependence on the film thickness at $4 \mathrm{~K}(\mathrm{~d})$. The inset indicates the spin coherence length. between measured (higher) and actual resistivity (lower), leading to uncertainty in $p$. Indeed, decreasing $\rho(47 \mathrm{~nm})$ by a moderate factor of 1.2 increases $p$ to 1.9 .

The extracted spin coherence lengths $L_{s o}$ are approximately 30,20 , and $30 \mathrm{~nm}$ (with $\sim 50 \%$ accuracy) for the 47 , 55 , and $76 \mathrm{~nm}$ thick films, respectively, and are independent of $T$ (similar to Ref. 11) in the low $T$ range investigated. $L_{s o}$ depends on the strength of SOI, on $L_{0}$ (in our experiments dominated by impurity scattering at low $T$ ), and on random SOI fluctuations. ${ }^{17}$ The former is a structural property while both the latter are independent of $T$ at low $T$, and hence no strong $T$ dependence is expected for $L_{s o}$ in our data. Within the accuracy of $L_{s o}$, a conclusive dependence on $t$ cannot be drawn. However, the data can, at best, suggest only a weak $t$ dependence, which is in contrast to the monotonic $t$ dependence of the mobility and $L_{0}$; thus $L_{0}$ may not be the appropriate length scale affecting $L_{s o}$ in our films. $L_{s o}$ can in some cases be independent of $L_{0}$ due to fluctuations in the SOI on a length scale smaller than $L_{0} \cdot{ }^{17}$ The strong SOI in our Bi films may carry fluctuations on a scale smaller than $L_{0}$ and may thereby limit $L_{s o}$ to be comparable or shorter than $L_{0}$, as observed in our experiments.

$L_{\phi}$ increases linearly with $t$. Films thicker than $76 \mathrm{~nm}$ did not exhibit WAL since the visibility $\Delta \rho / \rho \propto \rho / t$, and hence $L_{\phi}$ is inaccessible by WAL for films with $t>76 \mathrm{~nm}$. The $2 \mathrm{D}$ quantum coherence is a bulk carrier effect, denoting the regime where $L_{\phi}>t$, and $2 \mathrm{D}$ interference effects are observable even though the bulk electrons are kinematically 3D. An influence to $L_{\phi}$ from the surface states may also be present but cannot be distinguished in our data. The $76 \mathrm{~nm}$ film provides the optimal quantum coherence properties that can still be probed by 2D interference phenomena.

In conclusion, we present the deposition and properties of high quality Bi films suitable for patterning into quantum devices. The mobilities are thickness dependent and are consistent with values encountered in MBE grown Bi films. Our studies show that a $76 \mathrm{~nm}$ Bi film optimally exhibits bulklike properties and 2D quantum coherence phenomena. Analysis of WAL indicates a linear thickness dependence of $L_{\phi}$ and phonon dominated inelastic scattering above $2 \mathrm{~K}$. With high mobility Bi thin films, quantum coherent transport in the strong SOI regime can be studied to gain insight on SOI and Bi quantum devices.

We acknowledge funding from the Department of Energy through grant DOE DE-FG02-08ER46532.

${ }^{1}$ P. Kapitza, Proc. R. Soc. London, Ser. A 119, 358 (1928).

${ }^{2}$ L. Shubnikov and W. J. de Haas, Proc. R. Acad. Sci. Amsterdam 33, 130 (1930).

${ }^{3}$ W. J. de Haas and P. M. van Alphen, Proc. R. Acad. Sci. Amsterdam 33, 680 (1930).

${ }^{4}$ D. Shoenberg, Proc. R. Soc. London, Ser. A 170, 341 (1939).

${ }^{5}$ V. S. Édel'man, Adv. Phys. 25, 555 (1976).

${ }^{6}$ C. R. Ast and H. Höchst, Phys. Rev. B 66, 125103 (2002).

${ }^{7}$ Y. M. Koroteev, G. Bihlmayer, J. E. Gayone, E. V. Chulkov, S. Blügel, P. M. Echenique, and P. Hofmann, Phys. Rev. Lett. 93, 46403 (2004).

${ }^{8}$ D. L. Partin, J. Heremans, D. T. Morelli, C. M. Thrush, C. H. Olk, and T. A. Perry, Phys. Rev. B 38, 3818 (1988).

${ }^{9}$ N. Marcano, S. Sangiao, C. Magén, L. Morellón, M. R. Ibarra, M. Plaza, L. Pérez, and J. M. De Teresa, Phys. Rev. B 82, 125326 (2010).

${ }^{10}$ B. Hackens, J. P. Minet, S. Faniel, G. Farhi, C. Gustin, J. P. Issi, J. P. Heremans, and V. Bayot, Phys. Rev. B 67, 121403 (2003). 
${ }^{11}$ M. Rudolph and J. J. Heremans, Phys. Rev. B 83, 205410 (2011).

${ }^{12}$ C. Li, Z. Q. Zeng, D. S. Fan, Y. Hirono, J. Wu, T. A. Morgan, X. Hu, S. Q. Yu, Z. M. Wang, and G. J. Salamo, App. Phys. Lett. 99, 243113 (2011).

${ }^{13}$ H. Hattab, E. Zubkov, A. Bernhart, G. Jnawali, C. Bobisch, B. Krenzer, M. Acet, R. Möller, and M. Horn-von Hoegen, Thin Solid Films 516, 8227 (2008).
${ }^{14}$ W. Shim, J. Ham, J. Kim, and W. Lee, Appl. Phys. Lett. 95, 232107 (2009).

${ }^{15}$ S. Hikami, A. Larkin, and Y. Nagaoka, Prog. Theor. Phys. 63, 707 (1980). ${ }^{16}$ E. Abrahams, P. Anderson, D. Licciardello, and T. Ramakrishnan, Phys. Rev. Lett. 42, 673 (1979).

${ }^{17}$ M. M. Glazov, E. Ya. Sherman, and V. K. Dugaev, Physica E 42, 2157 (2010). 